Published in final edited form as:

World Neurosurg. 2016 November ; 95: 406-413. doi:10.1016/j.wneu.2016.06.008.

\title{
Multimodal Navigation in Endoscopic Transsphenoidal Resection of Pituitary Tumors using Image-based Vascular and Cranial Nerve Segmentation: A Prospective Validation Study
}

\author{
Parviz Dolati, MD ${ }^{1}$, Daniel Eichberg, MD ${ }^{1}$, Alexandra Golby, MD¹, Amir Zamani, MD², and \\ Edward Laws, MD1 \\ ${ }^{1}$ Department of neurosurgery, Brigham and Women's Hospital, Harvard Medical School \\ ${ }^{2}$ Department of radiology, Brigham and Women's Hospital, Harvard Medical School
}

\section{Abstract}

Introduction-Transsphenoidal surgery (TSS) is a well-known approach for the treatment of pituitary tumors. However, lateral misdirection and vascular damage, intraoperative CSF leakage, and optic nerve and vascular injuries are all well-known complications, and the risk of adverse events is more likely in less experienced hands. This prospective study was conducted to validate the accuracy of image-based segmentation in localization of neurovascular structures during TSS.

Methods-Twenty-five patients with pituitary tumors underwent preoperative 3TMRI, which included thin-sectioned 3D space T2, 3D Time of Flight and MPRAGE sequences. Images were reviewed by an expert independent neuroradiologist. Imaging sequences were loaded in BrainLab iPlanNet (16/25 cases) or Stryker ( $9 / 25$ cases) image guidance platforms for segmentation and preoperative planning. After patient registration into the neuronavigation system and subsequent surgical exposure, each segmented neural or vascular element was validated by manual placement of the navigation probe on or as close as possible to the target. The audible pulsations of the bilateral ICA were confirmed using a micro-Doppler probe.

Results-Pre-operative segmentation of the ICA and cavernous sinus matched with the intraoperative endoscopic and micro-Doppler findings in all cases (Dice Similarity Coefficient $=1$ ). This information reassured the surgeons with regard to the lateral extent of bone removal at the sellar floor and the limits of lateral exploration. Excellent correspondence between image-based segmentation and the endoscopic view was also evident at the surface of the tumor and at the tumor-normal gland interfaces. This assisted in preventing unnecessary removal of the normal pituitary gland. Image-guidance assisted the surgeons in localizing the optic nerve and chiasm in $64 \%$ of the cases and the diaphragma sella in $52 \%$ of cases, which helped to determine the limits of upward exploration and to decrease the risk of CSF leakage. The accuracy of the measurements was $1.20+0.21 \mathrm{~mm}($ mean $+/-\mathrm{SD})$.

Conclusion-Image-based pre-operative vascular and neural element segmentation, especially with 3D reconstruction, is highly informative preoperatively and potentially could assist less experienced neurosurgeons in preventing vascular and neural injury during TSS. Additionally, the accuracy found in this study is comparable to previously reported neuronavigation measurements. This novel preliminary study is encouraging for future prospective intraoperative validation with larger numbers of patients. 


\section{Keywords}

Segmentation; Pituitary tumor; Optic nerve; Internal carotid artery; MRI; Navigation

\section{Introduction}

The fundamental aims of pituitary tumor surgery are to debulk or resect the tumor mass, normalize hormonal hypersecretion, and eliminate the potential for recurrence, while avoiding damage to normal surrounding structures ${ }^{1}$. The current consensus is that surgical treatment is required for the majority of symptomatic pituitary adenomas ${ }^{2}$. The diagnosis of a non-secreting, a hormonally inactive pituitary tumor with mass effect, a hormonally hypersecreting pituitary tumor resistant to medical management, tumor-associated hypopituitarism, and unsuccessful prior therapy are general indications for surgery ${ }^{2-9}$. Additionally, the most rapid and reliable relief from optic nerve and chiasmal compression is achieved by surgery, which is particularly beneficial if severe loss of vision occurred acutely, as in pituitary tumor apoplexy ${ }^{10,11}$. Although the effectiveness of conventional microsurgical approaches to transsphenoidal surgery (TSS) in the treatment of pituitary tumors is well established $^{9}$, the line of sight and anatomical visualization is limited by the narrowness of the surgical corridor of the approach ${ }^{12}$. Endoscopic TSS can often offer improved visibility compared to traditional microsurgery.

Advances in pituitary surgery have involved the implementation of intraoperative neuronavigation systems, which enable enhanced visualization of adjacent neural, vascular, and ventricular structures. The most commonly used procedure for intra-operative imaging and navigation had been radio-fluoroscopic control ${ }^{2}$, but intra-operative MRI and 2dimensional high-resolution ultrasound, have also been used for neuronavigation. Although potentially effective, these techniques are not widely available.

The current prospective study was conducted to validate the accuracy of image-based segmentation in localization of neurovascular structures linked with frameless stereotactic navigation during TSS and to investigate its feasibility and benefits of this approach.

\section{PATIENTS AND METHODS}

\section{Study Size and Design}

This was a prospective study and the primary endpoints were defined as correspondence of pre-operative segmentation of the ICA, cavernous sinus, surface of the tumor, and tumornormal gland interfaces with the intra-operative endoscopic and micro-Doppler findings, as well as the status of pituitary gland position and success of localization of the optic nerve, optic chiasm, and diaphragm sella.

A total of 25 patients with sellar lesions (23 pituitary tumors of various subtypes and sizes, 1 dermoid tumor, and 1 arachnoid cyst) were enrolled in this study at a single institution from January to June 2014 (table 1). These patients underwent preoperative 3TMRI and MRA, which included thin-sectioned 3D space T2, 3D Time of Flight and MPRAGE sequences and Time of flight images MR angiography (TOF). An independent neuroradiologist reviewed 
the images. Imaging sequences were loaded in BrainLab iPlanNet (BrainLab AG, Munich, Germany) (16/25 cases) or Stryker (Stryker Corporation, Kalamazoo, Michigan, USA) (9/25 cases) neuronavigation platforms for segmentation and pre-op planning. The type of system applied was based on availability. For each patient, image-based pre-operative vascular (cavernous sinus, ICA and its branches) and neural element (optic nerve, chiasm, and infundibulum) segmentation with 3D reconstruction was performed. These image fusion studies and segmentations were utilized intraoperatively during real time neuronavigation. Data were prospectively collected and reviewed after approval by the Hospital Institutional Review Board.

Preoperative patient's Registration-After surgical positioning, the patient's head was registered to the intra-op neuronavigation system. In cases using BrainLab system for navigation, our registration was based on surface matching covering the skull base and most of the calvaria. In cases navigated using the Stryker system, we used the facemask for registration to obtain optimal accuracy. Subsequently, after registration and surgical exposure, each segmented neural or vascular element was validated by manual placement of the navigation probe directly on that object or as closely as possible to the target.

Intra-operative Navigation-The midline bony landmarks, as they are fixed in position, were used as verification points as the exposure and necessary bone removal were performed. Upon reaching the sellar floor, the anterior, posterior and lateral margins were checked. After removing the sellar floor, the surface of the dura was checked in different locations: at the center, anterior, posterior and lateral edges to determine how far the tumor was located from the dural surface, and to choose a point for dural opening for optimal access to the tumor.

For the cavernous sinuses, intraoperative localization was performed where the segmentation indicated the location of medial wall of cavernous sinus. This was confirmed all along both lateral gutters. We compared this with visual inspection of these lateral margins under endoscopic visualization and confirmed the sinuses. We recorded the differences in amount of bone removal as the accuracy of the system.

For the carotid arteries, due to their location inside the cavernous sinus, it is not possible to put the navigation probe on their surface unless tumor has invaded the sinus and the carotid arteries are encased. Therefore, the medial edge of the cavernous sinus (the lateral edge of our surgical field) was checked also for the carotid artery audible pulses using a microDoppler to determine the accuracy of the navigation data. The dural opening was limited accordingly.

For the optic nerves, we checked their location against the navigation data set by placing the navigation probe at the deepest part of our field before and after resection of the tumor. In large tumors, by navigation and eventually direct visualization of the optic nerves after decompression, the location of the optic nerves could be directly confirmed. Also, checking around the optic canal in some cases was helpful. In localizing the optic nerves, we tried not to pass the sellar floor arachnoid unless this exposure was clinically needed in large tumors for decompression. 
Data Sources: Correspondence of pre-operative segmentation of the ICA, cavernous sinus, surface of the tumor, and tumor-normal gland interfaces with the intra-operative endoscopic and micro-Doppler findings was determined by the surgeons at the time of surgery and screen shots were captured and recorded. The extent of pituitary tumor resection and success of localization of the optic nerve, optic chiasm, and diaphragm sella was also determined by the surgeons at the time of surgery. Tumor pathology was determined by an independent neuropathologist.

Statistical Methods: Patients' clinical and tumor characteristics (age, sex, presenting symptoms, tumor pathology, induction of anesthesia to incision time, incision to surgery end time, induction to surgery end time) were evaluated using the mean and range for continuous variables and the frequency count for categorical factors. The Dice similarity coefficient index was used as the spatial overlap index to compare the similarity of the pre-operative segmentation of the ICA, cavernous sinus, optic nerve and chiasm, and diaphragm sella with the intra-operative endoscopic and micro-Doppler findings.

\section{RESULTS}

\section{A-Descriptive Data}

Participants

The mean age was 47.7 years, and there were 14 male and 11 female patients. The most common presenting symptom was visual deficits in 10 patients (40\%), acromegaly and visual field deficits in 7 patients (28\%), Cushing's disease in 3 patients (12\%), hyperthyroidism in 1 patient (4\%), and progressive visual deficit and endocrine disorder in 4 patients (16\%) (table 1).

Tumor Pathology-Twelve patients (48\%) had null cell pituitary macroadenoma ( 8 primaries and 4 recurrences), 7 patients (28\%) had growth hormone-secreting macro- or micro-adenoma, 3 patients (12\%) had ACTH-secreting microadenomas, 1 patient (4\%) had a dermoid tumor, 1 patient (4\%) had a TSH-secreting microadenoma, and 1 patient (4\%) had an intra-sellar arachnoid cyst (table 1).

\section{B-Operative Data}

The mean time from completion of induction of anesthesia to incision was 31.3 minutes (range 16 minutes to 47 minutes), from incision to the end of surgery was 142.8 minutes (range 98 minutes to 209 minutes), and from the induction of anesthesia to the end of surgery was 174.2 minutes (114 minutes to 239 minutes). No major intraoperative bleeding or significant changes in patients' vital signs were reported. Patients' registration to the neuronavigation systems was satisfactory in all cases. At each stage, by placing the navigation probe over the fixed bony landmarks, any misregistration was rolled out.

As is evident in figures 1.A and 1.B, after fusion of all pre-operative images on BrainLab iPlanNet or Stryker neuronavigation planning software, the detail of the adenoma, the small hematoma inside the adenoma, adenoma's invasion into the cavernous sinus, medial margins of the cavernous sinus, course and location of the ICA in relation to the midline sellar floor 
and from the lateral edge of the adenoma, position of the pituitary stalk and posterior pituitary gland were all clearly visible.

As is seen in figure 1.C, excellent correspondence (Dice similarity coefficient $=1$ ) was found between image-based segmentation and endoscopic view at the surface of the tumor and tumor-normal gland interfaces. Navigation assisted to determine how far the tumor was located from the dural surface, and to choose a point for dural opening for optimal access to the tumor. Moreover, it assisted to limit especially the lateral margins of the dural opening before reaching into the cavernous sinus's medial margins.

Pre-operative segmentation of the ICA and cavernous sinus matched with the intra-operative endoscopic and micro-Doppler findings in all cases (Dice similarity coefficient $=1$ ). In other word, intraoperative surgical findings had complete anatomical correspondences with preoperative segmentations, which were utilized intraoperatively. As is seen in Figure 1.D, The $3 \mathrm{D}$ reconstruction of these fused data was also available during the surgery in all cases and assisted in faster and confident localization of the neurovascular elements. Injury to cavernous sinus wall or significant venous bleeding was not reported in any cases.

Figure 1.A and 1.B show that the optic chiasm and nerves, which are difficult to be visualized in pre-segmentation images, are clearly seen in post-segmentation images. The patient presented in figure 1 was suffering from visual field defect. As is obviously seen in figure 1.B, the optic chiasm was pushed superiorly and flattened by the pituitary macroadenoma. Both optic nerves were also pushed superiorly and laterally. Overall, considering the deep location of the optic nerves and chiasm, they are the most challenging elements to verify with navigation and direct visualization of these nerves is often not possible. However, by checking around the optic canal in some cases, and direct imageguided visualization of the optic nerves after resection of the large tumors in other cases, optic nerves and chiasm were successfully localized in 64\% of cases (Figure 1.E).

\section{C-Complications}

No vascular or cranial nerve injury was noted. Prolonged diabetes insipidus (DI) occurred in one case, transient diabetes insipidus occurred in one case, and transient cerebrospinal fluid leakage occurred in one case. DI was treated by DDAVP and the CSF leakage was managed by lumbar drainage of CSF.

\section{D-Clinical and Radiological Outcome}

In those patients who presented with visual decline, improvement in vision was noticed in 8 of 10 cases $(80 \%)$ after removal of the tumor and decompression of the optic apparatus. Transient CSF leakage in one patient was treated conservatively.

Gross total resection of the tumor was achieved in 22 patients (88\%) based on the postoperative MRI. Residual tumor was present in three cases, one extending into the cavernous sinus and two with extension beyond and posterior to the anterior communicating artery, were identified by post-operative MRI. These patients were considered for radiation therapy. 


\section{E-Accuracy}

For each landmark, the error of the neuronavigation system as compared to the anatomy was measured. The mean accuracy measured was $1.20+0.21 \mathrm{~mm}$ (mean + standard deviation).

\section{Discussion}

Our preliminary study shows that image-based pre-operative vascular and neural element segmentation, especially with 3D reconstruction, is highly informative for both preoperative planning and intraoperative guidance of microsurgical transsphenoidal surgery (TSS). Preoperative 3D segmentation can help the surgeon to understand the relationship of the tumor to surrounding neurovascular structures (Figures 1-A and 1-B).

Three-dimensional reconstruction of imaging data during endoscopic TSS has previously been reported ${ }^{13}$. Although that study found that the image-based reconstructed picture correlated adequately with the intraoperative endoscopic view, the group did not report the intraoperative accuracy of measurements ${ }^{13}$, preventing objective comparison with similar investigations.

Neuronavigation only provides surgical utility if the image guidance system data accurately correspond to the intraoperative patient anatomy. A retrospective review of the literature found that mean neuronavigation accuracies have ranged from 1.8 to $5 \mathrm{~mm}^{14-16}$. Recently, one group has improved on this accuracy with the use of preoperatively acquired 3D digital subtraction angiography (DSA) registered by the facial surface anatomy contained within the data set ${ }^{17}$. The investigators validated their novel neuronavigation technique in a cadaver model, and reported an accuracy of $0.71 \pm 0.25 \mathrm{~mm}$ (mean $\pm \mathrm{SE})^{17}$. This accuracy may be overestimated, as the brain tissue and cerebrovasculature of their cadaver model were relatively anchored in place due to formalin fixation and injection of the vasculature with latex. Thus, the accuracy-degrading complication of intraoperative brain shift was largely eliminated. Although DSA remains the gold standard for imaging of cerebrovascular pathology due to its superior detection sensitivity and spatial resolution over MR angiography and $\mathrm{CT}$ angiography ${ }^{18}$, the imaging modality is not without its limitations and risks. DSA offers high spatial resolution of the cerebral vasculature, however, it provides only limited anatomical information about extravascular structures, knowledge of which may minimize surgical morbidity and mortality. Additionally, DSA is a costly and invasive procedure which confers a risk of permanent neurologic complication in $0.1 \%$ to $0.5 \%$ cases $^{19-23}$.

Our group achieved a measurement accuracy of $1.20+0.21 \mathrm{~mm}$ (mean $+\mathrm{SD})$, which is comparable to previously reported accuracies ranging from 0.71 to $5 \mathrm{~mm}^{14,17,24,25}$. Additionally, we utilized preoperative 3TMRI for image acquisition, which is a far less invasive procedure than DSA.

These results were consistent with our group's prior experience with preoperative 3D segmentation. Image-based vascular and cranial nerve 3D segmentation was found to have an accuracy of $0.45+0.21 \mathrm{~mm}$ (mean + standard deviation) for microvascular decompression, with excellent correspondence found between image-based segmentation and endoscopic and microscopic images and videos ${ }^{24}$. Additionally, 3D reconstruction was 
found to have an accuracy of $0.45+0.21 \mathrm{~mm}$ (mean + standard deviation) for skull base tumor resection, and was highly informative preoperatively and could increase the vigilance of neurosurgeons for preventing neurovascular injury during skull base operations ${ }^{25}$.

Advances in image technology resulting in improvement of spatial resolution have tremendously improved preoperative visualization of the cranial nerves. These delicate imaging schemes like 3Tesla MRI/ Fast imaging employing steady-state acquisition (FIESTA) and Three-dimensional (3D) constructive interference in steady state (CISS) sequences, are able to demonstrate details of small anatomic structures which can be very informative before attacking a tumor or other lesion in the sellar and supra sellar regions. Using these images, we can see very small cranial nerves passing in the cisterns or in close vicinity to a tumor. However, in the current study and our previous reports ${ }^{24}, 25$, we showed how we can upload these images into the navigation system, merge them together, segment cranial nerves and the tumor or adjacent blood vessels and use them in real time during the operation to prevent damage to neurovascular elements. Therefore, the major advantage of our method is actual clinical application of these images in the operating room and inside the cranium.

Our study has shown that image-based pre-operative vascular and neural element segmentation with 3D reconstruction was highly informative regarding the lateral extent of bone removal at the sellar floor and the limits of lateral explorations, as pre-operative segmentation of the ICA and cavernous sinus matched with the intra-operative endoscopic and micro-Doppler findings in all cases. One of the major values of the preoperative segmentation is pre-operative evaluation and prediction of the cavernous sinus involvement and carotid artery encasement, as shown in figure 1-B. In this case, it was difficult to discern this relationship in non-segmented images, however, after segmentation and especially after rending as 3D images, one can see that the tumor has invaded the cavernous sinus border. Especially in cases of cavernous sinus involvement, pre-operative review of these segmented and fused images can forewarn the surgeons of possible bleeding as a result of cavernous sinus invasion and could be extremely helpful for protection of the ICA during tumor resection.

Additionally, this technique aided in preventing unnecessary removal of the normal pituitary gland, as there was excellent correspondence between image-based segmentation and endoscopic view at the surface of the tumor and tumor-normal gland interfaces (Figure. 1.C). Image-based segmentation was also beneficial in determining the limits of upward exploration and decreasing the risk of CSF leakage. Moreover, image-guidance allowed the surgeons to localize the optic nerve and chiasm in $64 \%$ of cases and diaphragm sella in $52 \%$ of cases (Figure 1.E).

The major limitation of our study was the small number of cases included. The limited number of cases limits interpretation of patient complication rates, as well as determining how frequently image-based segmentation aids in identification of critical structures. It is our hope however, that the instructive cases presented here will inspire further research into this area. 
Image-based pre-operative vascular and neural element segmentation with 3D reconstruction is a promising intra-operative guidance tool, which may also be helpful for instruction and training.

\section{Conclusion}

Image-guided endoscopic trans-sphenoidal surgery provides a three-dimensional image for the surgeon, allowing for greater visual accuracy and surgical precision and a less time consuming procedure without radiation exposure or the need for additional personnel. Image-based pre-operative vascular and neural element segmentation, especially with 3D reconstruction, is highly informative preoperatively and could help young and inexperienced neurosurgeons to avoid vascular and neural injury during trans-sphenoidal surgeries, as well as providing reassurance to more experienced surgeons. Additionally, the accuracy reported in this study is comparable to previously reported neuronavigation measurements. Although the number of cases is too small to determine if pre-operative image-based segmentation of neurovascular structures reduces patient complication rates, it is our hope that this novel preliminary study is encouraging for future prospective intraoperative validation with larger numbers of patients.

\section{References}

1. Laws ER. Pituitary surgery. Endocrinol Metab Clin North Am. 1987; 16(3):647-665. [PubMed: 3319597]

2. Buchfelder M, Schlaffer S. Surgical treatment of pituitary tumours. Best Pract Res Clin Endocrinol Metab. 2009; 23(5):677-692. [PubMed: 19945031]

3. Buchfelder M. Treatment of pituitary tumors: surgery. Endocrine. 2005; 28(1):67-75. [PubMed: 16311412]

4. Nomikos P, Buchfelder M, Fahlbusch R. The outcome of surgery in 668 patients with acromegaly using current criteria of biochemical 'cure'. Eur J Endocrinol. 2005; 152(3):379-387. [PubMed: 15757854]

5. Chen JC, Amar AP, Choi S, Singer P, Couldwell WT, Weiss MH. Transsphenoidal microsurgical treatment of Cushing disease: postoperative assessment of surgical efficacy by application of an overnight low-dose dexamethasone suppression test. J Neurosurg. 2003; 98(5):967-973. [PubMed: 12744355]

6. Nomikos P, Ladar C, Fahlbusch R, Buchfelder M. Impact of primary surgery on pituitary function in patients with non-functioning pituitary adenomas -- a study on 721 patients. Acta Neurochir (Wien). 2004; 146(1):27-35. [PubMed: 14740262]

7. Webb SM, Rigla M, Wägner A, Oliver B, Bartumeus F. Recovery of hypopituitarism after neurosurgical treatment of pituitary adenomas. J Clin Endocrinol Metab. 1999; 84(10):3696-3700. [PubMed: 10523016]

8. Laws ER, Piepgras DG, Randall RV, Abboud CF. Neurosurgical management of acromegaly. Results in 82 patients treated between 1972 and 1977. J Neurosurg. 1979; 50(4):454-461. [PubMed: 423000]

9. Laws ER, Fode NC, Redmond MJ. Transsphenoidal surgery following unsuccessful prior therapy. An assessment of benefits and risks in 158 patients. J Neurosurg. 1985; 63(6):823-829. [PubMed: 2997414]

10. Powell M. Recovery of vision following transsphenoidal surgery for pituitary adenomas. $\mathrm{Br} \mathbf{J}$ Neurosurg. 1995; 9(3):367-373. [PubMed: 7546358]

11. Laws ER, Trautmann JC, Hollenhorst RW. Transsphenoidal decompression of the optic nerve and chiasm. Visual results in 62 patients. J Neurosurg. 1977; 46(6):717-722. [PubMed: 859014] 
12. Vitaz TW, Inkabi KE, Carrubba CJ. Intraoperative MRI for transphenoidal procedures: short-term outcome for 100 consecutive cases. Clin Neurol Neurosurg. 2011; 113(9):731-735. [PubMed: 21889838]

13. Wolfsberger S, Neubauer A, Bühler K, et al. Advanced virtual endoscopy for endoscopic transsphenoidal pituitary surgery. Neurosurgery. 2006; 59(5):1001-1009. discussion 1009-1010. [PubMed: 17143234]

14. Stieglitz LH, Fichtner J, Andres R, et al. The silent loss of neuronavigation accuracy: a systematic retrospective analysis of factors influencing the mismatch of frameless stereotactic systems in cranial neurosurgery. Neurosurgery. 2013; 72(5):796-807. [PubMed: 23334280]

15. Elias WJ, Chadduck JB, Alden TD, Laws ER. Frameless stereotaxy for transsphenoidal surgery. Neurosurgery. 1999; 45(2):271-275. discussion 275-277. [PubMed: 10449071]

16. Lasio G, Ferroli P, Felisati G, Broggi G. Image-guided endoscopic transnasal removal of recurrent pituitary adenomas. Neurosurgery. 2002; 51(1):132-136. discussion 136-137. [PubMed: 12182410]

17. Stidd DA, Wewel J, Ghods AJ, et al. Frameless neuronavigation based only on 3D digital subtraction angiography using surface-based facial registration. J Neurosurg. 2014; 121(3):745750. [PubMed: 25036204]

18. Anxionnat R, Bracard S, Ducrocq X, et al. Intracranial aneurysms: clinical value of 3D digital subtraction angiography in the therapeutic decision and endovascular treatment. Radiology. 2001; 218(3):799-808. [PubMed: 11230659]

19. Kaufmann TJ, Huston J, Mandrekar JN, Schleck CD, Thielen KR, Kallmes DF. Complications of diagnostic cerebral angiography: evaluation of 19,826 consecutive patients. Radiology. 2007; 243(3):812-819. [PubMed: 17517935]

20. Heiserman JE, Dean BL, Hodak JA, et al. Neurologic complications of cerebral angiography. AJNR Am J Neuroradiol. 1994; 15(8):1401-1407. discussion 1408-1411. [PubMed: 7985557]

21. Dion JE, Gates PC, Fox AJ, Barnett HJ, Blom RJ. Clinical events following neuroangiography: a prospective study. Stroke. 1987; 18(6):997-1004. [PubMed: 3686597]

22. Earnest F, Forbes G, Sandok BA, et al. Complications of cerebral angiography: prospective assessment of risk. AJR Am J Roentgenol. 1984; 142(2):247-253. [PubMed: 6198889]

23. Waugh JR, Sacharias N. Arteriographic complications in the DSA era. Radiology. 1992; 182(1): 243-246. [PubMed: 1727290]

24. Dolati P, Golby A, Eichberg D, et al. Pre-operative image-based segmentation of the cranial nerves and blood vessels in microvascular decompression: Can we prevent unnecessary explorations? Clin Neurol Neurosurg. 2015; 139:159-165. [PubMed: 26476700]

25. Dolati P, Gokoglu A, Eichberg D, Zamani A, Golby A, Al-Mefty O. Multimodal navigated skull base tumor resection using image-based vascular and cranial nerve segmentation: A prospective pilot study. Surg Neurol Int. 2015; 6:172. [PubMed: 26674155] 


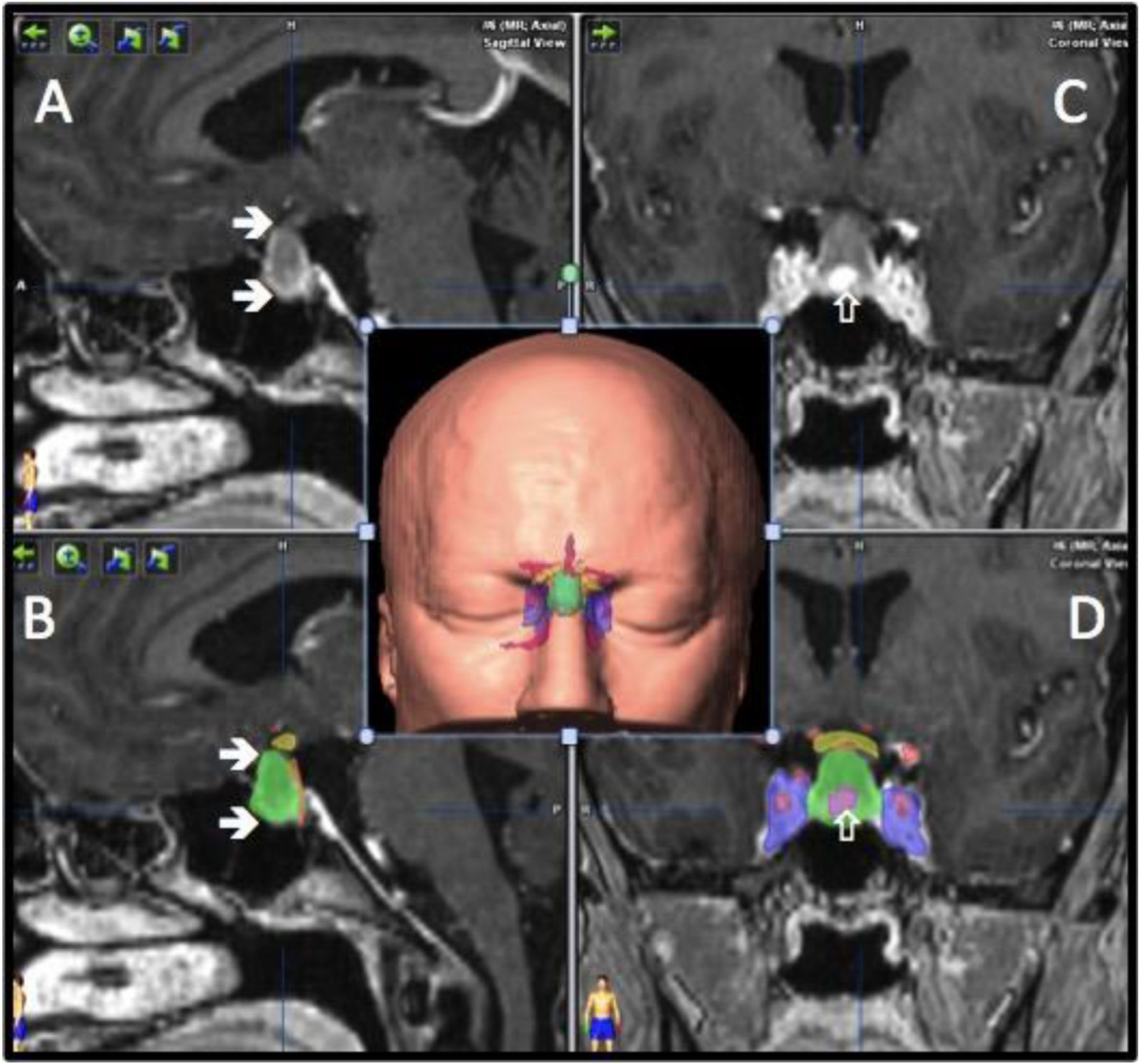

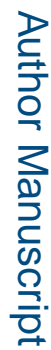




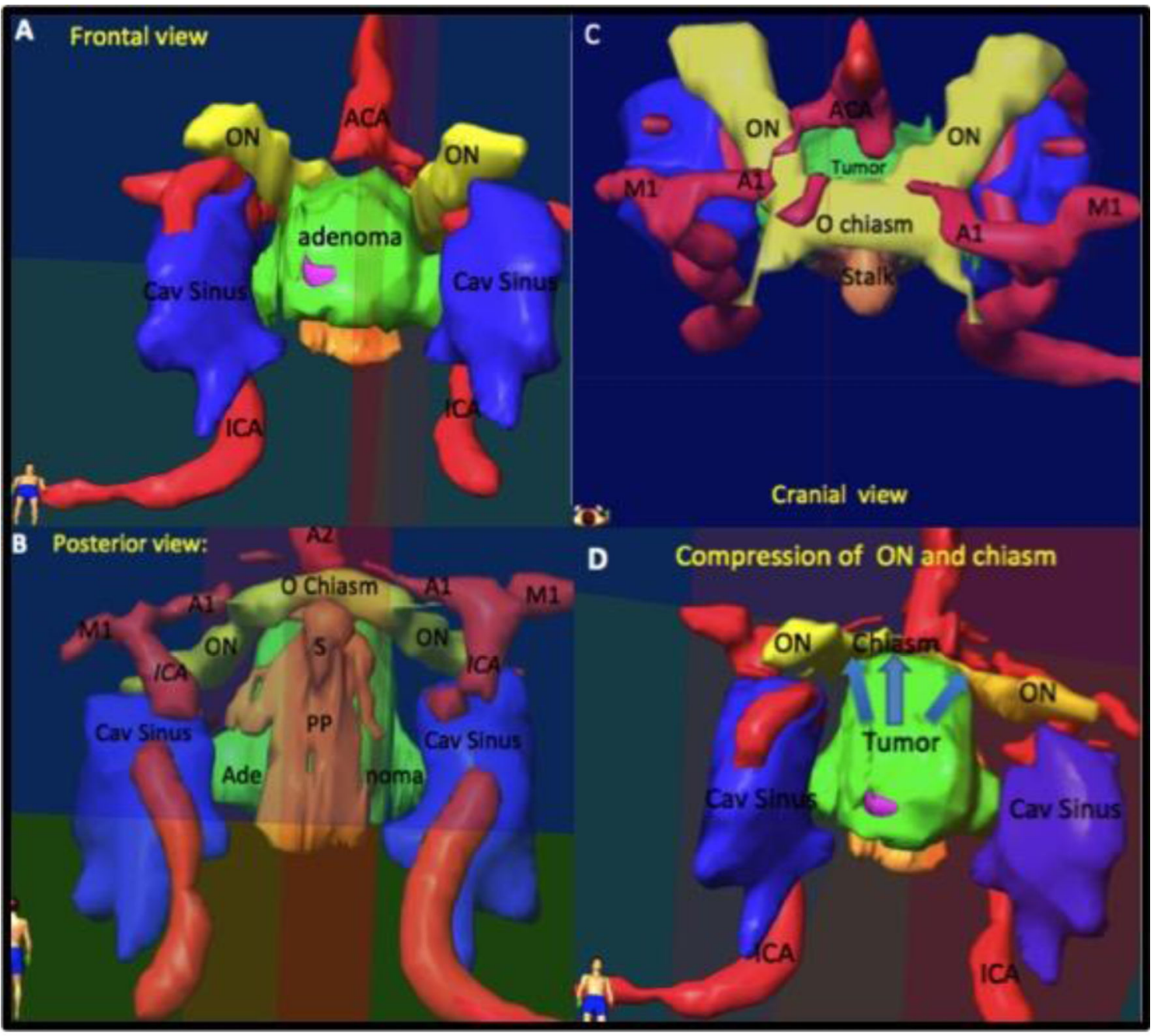

Abbreviations: ICA: Internal Carotid Artery, ACA, Anterior Cerebral Artery, A2: second segment of the Anterior Cerebral artery, M1: the first segment of the Middle Cerebral Artery ON: Optic Nerve, O Chiasm: Optic Chiasm, T: Tumor, Cav sinus: Cavernous Sinus (CS), DS: Dural Surface, S: stalk, PP: Posterior Pituitary, Nav probe: Navigation Probe 


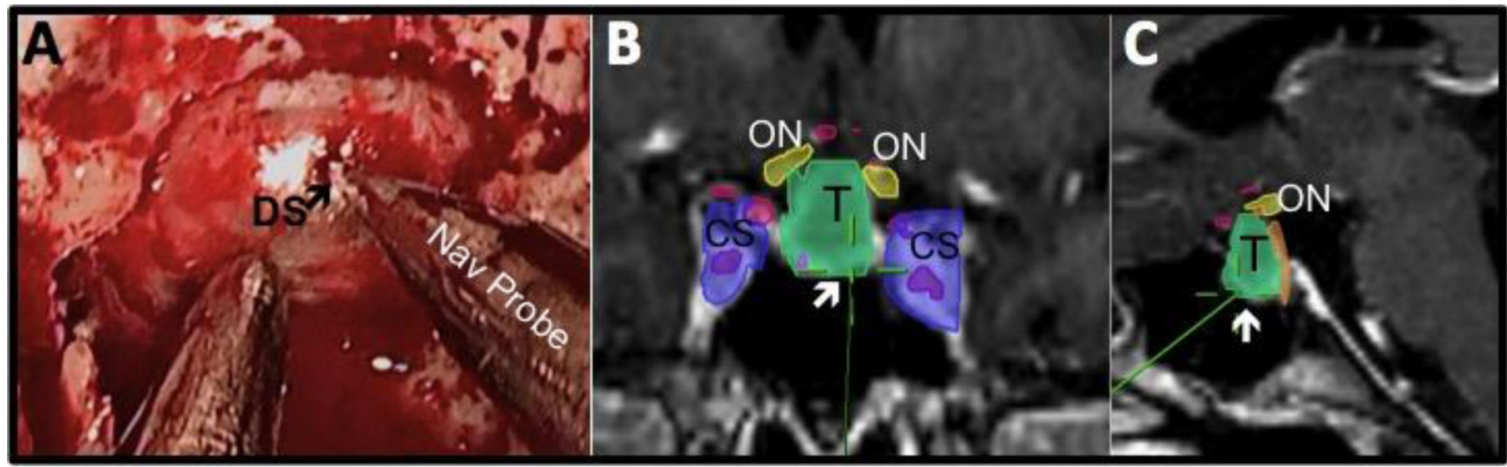

Abbreviations: CS: cavernous sinus, DS: dural surface, ON: optic nerve, T: tumor

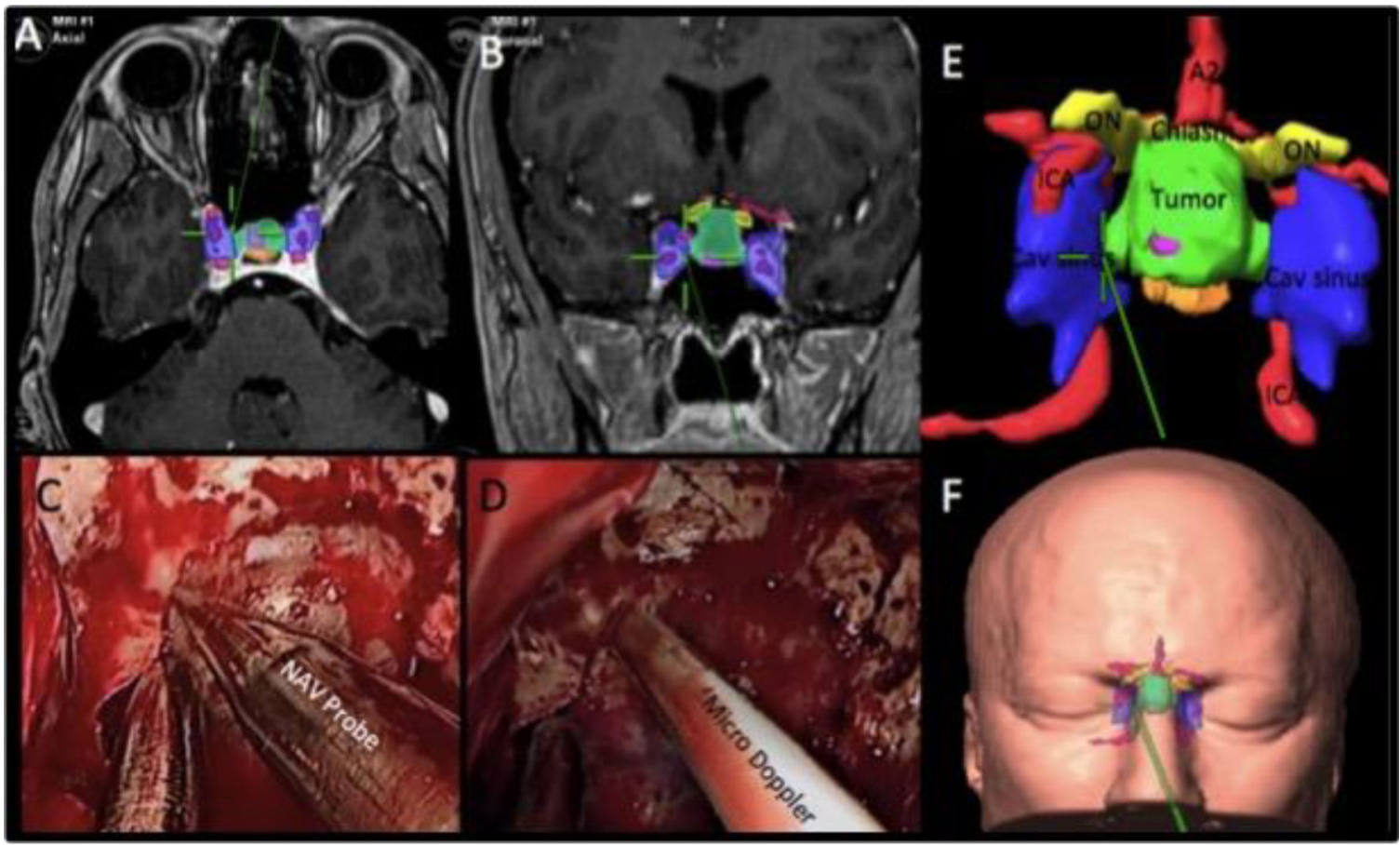

Abbreviations: A2: second segment of the Anterior Cerebral Artery, Cav sinus: cavernous sinus, ICA: internal carotid artery, ON: optic nerve 


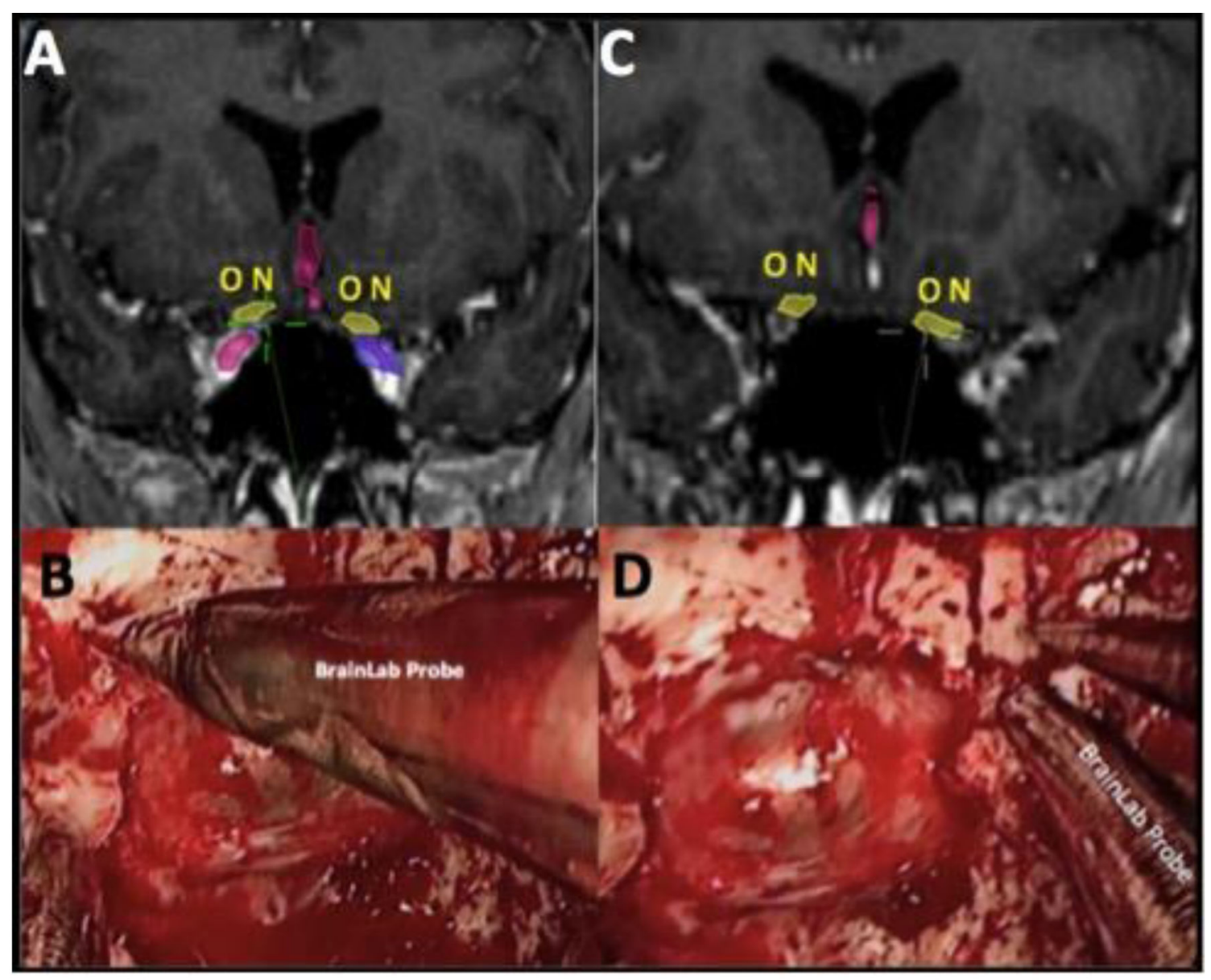

Abbreviations: ON: optic nerve

Abbreviation:

ICA: Internal Carotid Artery, ACA, Anterior Cerebral Artery, A2: second segment of the Anterior Cerebral artery, M1: the fist segment of the Middle Cerebral Artery ON: Optic Nerve, Chiasm: Optic Chiasm, T: Tumor, Cav sinus: Cavernous Sinus (CS), DS: Dural Surface, S: stalk, PP: Posterior Pituitary, Nav probe: Navigation Probe

Fig 1.

A 55 year-old man presented with headaches, double vision, and bitemporal hemianopsia. His brain MRI with gadolinium showed a pituitary macroadenoma.

Fig 1.A. Preoperative Non-segmented and segmented brain MRI.

Panel A (post Gadolinium sagittal brain MRI, white arrows) and panel C (post Gadolinium coronal brain MRI with area of bleeding, empty arrow). Patient's brain MRI was uploaded into the BrainLab and underwent pre-operative segmentation (panels B\&D) of the tumor (green), cavernous sinus (blue), optic nerve (yellow), internal carotid arteries (red). 
Fig. 1.B. Tri-dimensional reconstruction of the segmented areas of patient's sellar and parasellar region. Panel A, frontal view, showing the position of tumor (green) in relation to the cavernous sinus (blue, Cav Sinus), Internal carotid arteries (red, ICA), Optic nerves (yellow, $\mathrm{ON}$ ), Anterior cerebral arteries (red, ACA). The adenoma shows some extension into the cavernous sinus. Panel C, cranial view, showing flattening of the optic chiasm (o chiasm) and optic nerves and position of the pituitary stalk (S), first segments of the ACA (A1) and middle cerebral artery (M1). Panel B is the posterior view showing the position of the pituitary stalk (S), posterior pituitary (pink, PP) and adenoma. Finally, panel D is the transsphenoidal view, showing surgical corridor and compression of the optic nerves and chiasm by the macroadenoma. This $3 \mathrm{D}$ reconstruction was utilized during neuronavigation in a real time fashion (Fig 1.D, panel F) and the accuracy was checked by anatomical observation and micro Doppler finding.

Fig 1.C. Operative view corresponds to the surface of the tumor.

Panel A showing sellar floor after removal of the thin bone. Dural surface (DS, black arrow) was touched by navigation probe (Nav Probe) in different locations to define the center and lateral borders. Panels B and C are coronal and sagittal views and the green line is the representative of the Nav probe and the small white arrows are pointing to the contact point. Fig 1.D. Intra-operative validation of image-based segmentation in localizing the ICA and cavernous sinus using the micro-Doppler probe.

Panels A and B are the axial and coronal brain MRI during intraoperative navigation for determining tumor-cavernous sinus junction and validation of ICA. Panel $\mathrm{C}$ is the corresponding Nav probe (navigation probe) in the right lateral border of the tumor. Panel D, is showing the micro Doppler in the same location as the Nav probe to confirm the location of the ICA by listening to the audible internal carotid artery sound. Panels E and F are the 3D objects after segmentation, which are used for intraoperative navigation.

Fig 1.E. Correspondence of segmented images used for intraoperative navigation with operative view of optic nerves.

Panels $A \& B$ and $C \& D$ are the correspondence of intraoperative navigation of the right and left optic nerves $(\mathrm{ON})$, respectively. 


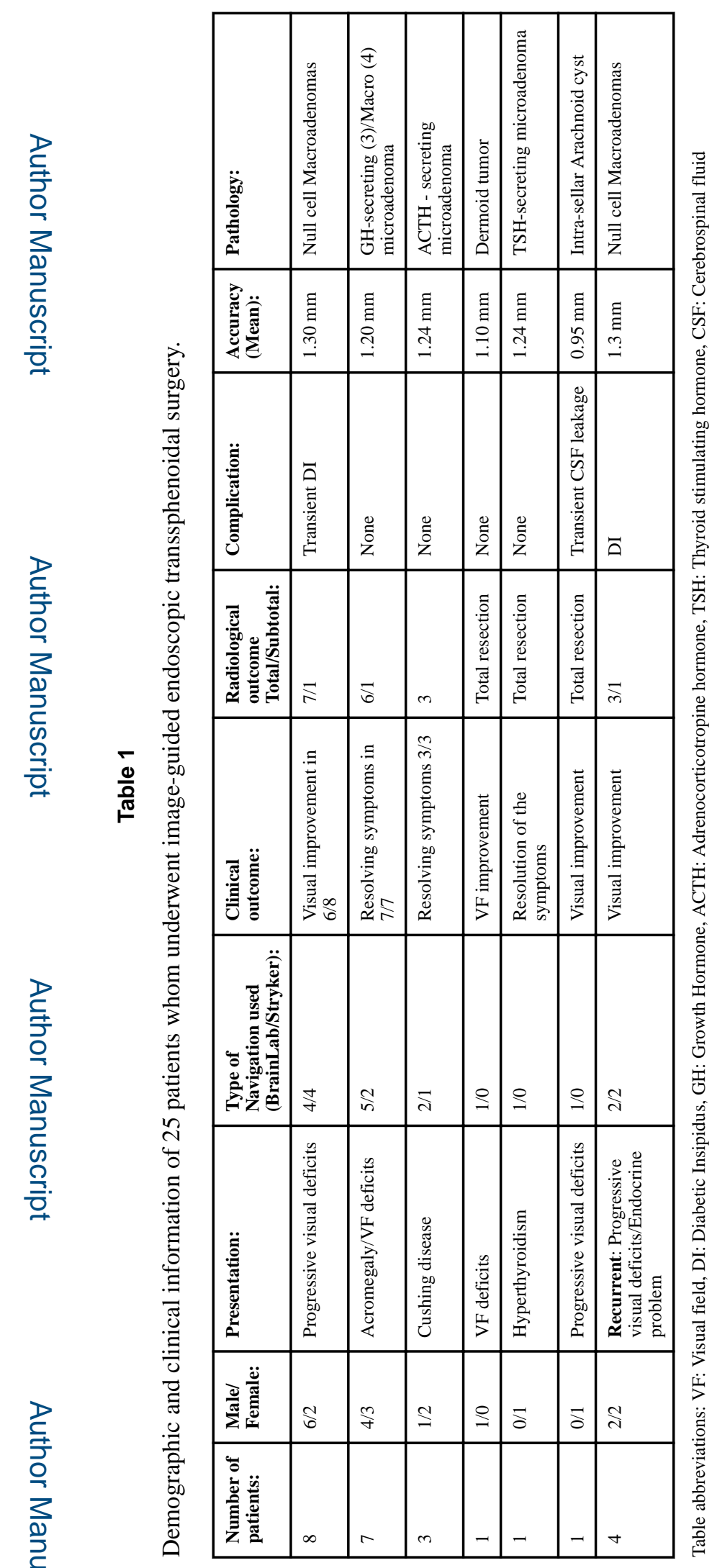

World Neurosurg. Author manuscript; available in PMC 2017 November 01. 